\title{
In vivo RAF signal transduction as a potential biomarker for sorafenib efficacy in patients with neuroendocrine tumours
}

\author{
M Quintela-Fandino ${ }^{1,2}$, M Krzyzanowska ${ }^{1}$, G Duncan ${ }^{3}$, A Young $^{3}$, M J Moore ${ }^{1}$, E X Chen ${ }^{1}$, A Stathis ${ }^{1}$, \\ R Colomer ${ }^{2}$, J Petronis ${ }^{1}$, M Grewal ${ }^{1}$, S Webster ${ }^{1}$, L Wang ${ }^{1}$ and L L Siu ${ }^{*}, 1$ \\ ${ }^{1}$ Division of Medical Oncology and Hematology, Princess Margaret Cancer Centre, University Health Network, University of \\ Toronto, 610 University Avenue, Suite 5-718, Toronto, Ontario, Canada M5G2M9; ${ }^{2}$ CNIO - Spanish National Cancer Research \\ Center Clinical Program, Melchor Fernandez Almagro 3, Madrid 28029, Spain and ${ }^{3}$ Department of Medical Biophysics, Ontario \\ Cancer Institute, University of Toronto, 620 University Avenue, Toronto, Ontario, Canada M5G2C9
}

Background: Targeted therapies elicit anticancer activity by exerting pharmacodynamic effects on specific molecular targets. Currently, there is limited use of pharmacodynamic assessment to guide drug administration in the routine oncology setting.

Methods: We developed a phosphoshift (pShift) flow cytometry-based test that measures RAF signal transduction capacity in peripheral blood cells, and evaluated it in a phase II clinical trial of oral sorafenib plus low-dose cyclophosphamide in patients with advanced neuroendocrine tumours (NETs), in order to predict clinical course and/or guide individual dose-titration.

Results: Twenty-two patients were enrolled. Median progression-free survival (PFS) was 3 months (95\% Cl 2-10.7), and one patient had a partial response. PFS was longer among five patients who demonstrated an increase in pShift after 7 days of sorafenib compared with those who did not (14.9 months vs 2.8 months; $P=0.047)$. However, pShift did not add value to toxicity-based dose-titration.

Conclusion: The pharmacodynamic assessment of RAF transduction may identify selected patients with advanced NETs most likely to benefit from the combination of sorafenib plus cyclophosphamide. Further investigation of this test as a potential biomarker is warranted.

The administration of targeted anticancer therapies can be optimised by the identification of predictive biomarkers that segregate responders from non-responders, and the evaluation of pharmacodynamic biomarkers that provide functional proof-ofmechanism (Gutierrez et al, 2009). Pharmacodynamic effects reported in many clinical trials successfully demonstrate target engagement and modulation, but their translation to clinical efficacy has been elusive, due in part to the dynamic nature of cellular signal transduction events. Nolan and colleagues (Irish et al, 2004; Sachs et al, 2005) proposed that the dynamic assessment of a signalling pathway in both the resting and stimulated conditions can be more informative than the resting condition alone. Thus, the phosphorylation status of the substrate of a targeted kinase may not reflect the actual pharmacodynamics of a drug if it is measured in fixed paraffinised tumour tissue, and alternative methodologies should be tested. As the antitumour efficacy of a drug is mediated by its pharmacodynamic effect, we hypothesised that determining the signal transduction in fresh tissue from patients undergoing treatment may help identify those most likely to benefit. As solid tumour tissue cannot be readily assessed in real-time due to the technical challenges in performing signalling pathway stimulation, we focused in using whole blood, which is another source of body tissue that is more accessible and can be stimulated ex vivo. 
Antiangiogenic agents have shown activity in neuroendocrine tumours (NET) (Raymond et al, 2011). In addition, the concurrent blockade of vascular endothelial growth factor receptor (VEGFR) and platelet-derived growth factor receptor (PDGFR) had been suggested to yield synergistic antitumour effects in a genetically engineered mouse model of NET when added to metronomic chemotherapy (Hanahan, 1985; Pietras and Hanahan, 2005) In these studies, four different therapeutic strategies, either alone or in combination, were tested for their efficacy in the NET model: PDGFR-B blockade, VEGFR2 blockade, low-dose continuous administration (metronomic) of cyclophosphamide, and a strategy termed 'chemo-switch' (consisting of starting cyclophosphamide at high doses and switching to metronomic administration after a few courses). Although the combination of the four strategies yielded the highest effect, we felt that implementing the chemo-switch strategy in the clinical setting would be challenging, thus we opted to evaluate the combination of the first three strategies, all of which were also highly effective in the model. In order to explore such an approach in advanced NET patients, we conducted a phase II clinical trial combining the multikinase inhibitor sorafenib, that blocks VEGFR, PDGFR and RAF isoforms (Carter, 2010), with low-dose cyclophosphamide.

The end points of the trial included the evaluation of efficacy, as well as the pharmacodynamic effects of sorafenib in the peripheral blood mononuclear cells (PBMCs) compartment of whole blood. A flow cytometry-based assay was developed to assess in real-time, dynamically and quantitatively, the capacity of the RAF pathway to transduce a specific signal (interleukin 3 (IL3)-stimulation) in PBMCs, both in basal and stimulated states (Poulikakos et al, 2010; Quintela-Fandino et al, 2010). This phosphoshift assay (pShift) aimed to elucidate the effects of sorafnib on the RAF signal transduction capacity (RAF STC) in both states. RAF STC was chosen as the preferred parameter for our study for technical reasons. Although based on preclinical data, the potential activity of sorafenib in NETs is primarily due to its antiangiogenic activity (Pietras and Hanahan, 2005), sorafenib modulates RAF with a similar $K_{\mathrm{m}}$ as that of VEGFR2 or PDGFR-B (Carter, 2004). However, there are no available antibodies that recognise the phosphorylated form of VEGFR2 for flow cytometry and the expression of PDGFR-B is below the detection limit of this technique in PBMCs. RAF expression, however, is abundant in all PBMCs and downstream phospho-sites are easily detected by flow cytometry. Owing to the similar $K_{\mathrm{m}}$ values, we hypothesised that demonstrating RAF modulation upon sorafenib exposure would translate to VEGFR2 and PDGFR-B pharmacodynamic modulation as well.

The objectives of this trial were to determine the efficacy of individually dose-titrated sorafenib given in combination with metronomic cyclophosphamide in patients with advanced NET, and to ascertain the value of the pShift assay in guiding the titration of sorafenib, and as a potential prognostic/predictive biomarker of clinical outcome.

\section{PATIENTS, MATERIALS AND METHODS}

Eligibility criteria. Inclusion criteria were the following: pathologically confirmed, unresectable carcinoid, or islet cell carcinoma; Eastern Cooperative Oncology Group performance status of 0-2; unlimited prior systemic therapy except for sorafenib; mandatory documentation of progressive disease by RECIST 1.0 criteria (Therasse et al, 2000) within 6 months before study entry; life expectancy of $>3$ months; measurable disease; the absence of brain metastasis and $\geqslant 6$ weeks since the last chemotherapy/bland hepatic artery embolisation, chemotherapy, or other investigational agents. Adequate haematological function (absolute granulocyte count $\geqslant 1.5 \times 10^{9} 1^{-1}$, leukocyte count $\geqslant 3.0 \times 10^{9} 1^{-1}$, hemoglobin $\geqslant 100 \mathrm{gl}^{-1}$, and platelet count $\geqslant 100 \times 10^{9} 1^{-1}$ ), as well as adequate renal and hepatic functions (serum creatinine $\leqslant 1.5 \times$ upper limit of normal (ULN) or creatinine clearance $\geqslant 60 \mathrm{ml} \mathrm{min}^{-1}$, serum bilirubin $\leqslant 1.5 \times$ ULN and serum transaminases $\leqslant 2.5 \times$ ULN; up to $5 \times$ ULN if liver metastasis), and normal serum amylase/lipase levels were required.

All patients provided written informed consent. The study and its amendments were approved by the independent ethics committee/institutional review board at the Princess Margaret Hospital, Canada, and the study was conducted in accordance with the Declaration of Helsinki and Good Clinical Practice Guidelines.

Phosphoshift assay. The development, quantitation, flow cytometer settings, standardisation of pShift, test reproducibility, as well as results in healthy volunteers have been reported elsewhere (Quintela-Fandino et al, 2010). Phosphoshift measures the activation of the RAF signal transduction pathway by detecting the phosphorylation of MEK1/2 in cells unstimulated or stimulated using IL3, a specific activator of the RAF signalling pathway in normal monocytes (Yagisawa et al, 1999). A detailed experimental protocol is provided (Supplementary Material).

The assay is based on the following principles (Figure 1): (1) when RAF isoforms are stimulated in the absence of sorafenib, their STC is intact and thus capable of recruiting and phosphorylating MEK1/2, resulting in an increase in phospho-MEK1/2 signal (x-axis); (2) the difference between basal and stimulated phospho-MEK1/2 levels constitutes the 'phospho-shift' (pShift), which estimates RAF isoforms STC at a given moment; (3) RAF inhibitors such as sorafenib inhibit or stimulate transduction through the different RAF isoforms to a variable degree depending on the RAS/RAF genetic background (Rajakulendran et al, 2009; Hatzivassiliou et al, 2010; Poulikakos et al, 2010); (4) the comparison of pShift before and after exposure with a RAF inhibitor, reflects its pharmacodynamic effect on RAF isoforms STC. Detailed quantitation data are included in the Supplementary Material. Briefly, the test yields a numeric read-out that ranges from $-100 \%$ to $+100 \%$ to represent the change in STC from day 0 (R0) before starting sorafenib to day 7 on-treatment (R7), with negative and positive values denoting pShift inhibition and stimulation, respectively.

Study design and treatment regimen. This study was a prospective, phase II, open-label, single-arm, single-institution clinical trial to assess the efficacy of the combination of tailored sorafenib dosing plus metronomic cyclophosphamide in advanced, progressive NET (SORNET = Efficacy study of SORafenib and cyclophosphamide to treat NEuroendocrine Tumours, ClinicalTrials.gov trial registration ID: NCT00605566). The dose of sorafenib was to be titrated based on patients' toxicity and pShift value.

The study had a 'run-in' period in which the dose of sorafenib for the 'treatment phase' was established using three criteria based on toxicity, pShift and a maximum prespecified dose. The absence of haematologic grade 3 or intolerable non-haematologic grade 2 toxicities, a pShift inhibition of $<90 \%$, or a sorafenib daily dose of $<800 \mathrm{mg}$ b.i.d., as described in Figure 2, prompted a new doseescalation round of sorafenib. The first escalation round, from 200 to $400 \mathrm{mg}$ b.i.d., was an exception to these rules as it could occur regardless of the pShift value, as long as the toxicity criterion was not met. Dose-escalation of sorafenib aiming for pShift inhibition in this study was based on the results of a previous pilot study performed by our group evaluating pShift (Quintela-Fandino et al, 2010). In that study, seven patients were assayed and variable reductions of pShift values upon sorafenib exposure were observed but no patients demonstrated a pShift increase.

A baseline pShift test was determined on 'day R0', (defined as up to 7 days before the first dose of sorafenib). Subsequently, patients 
A
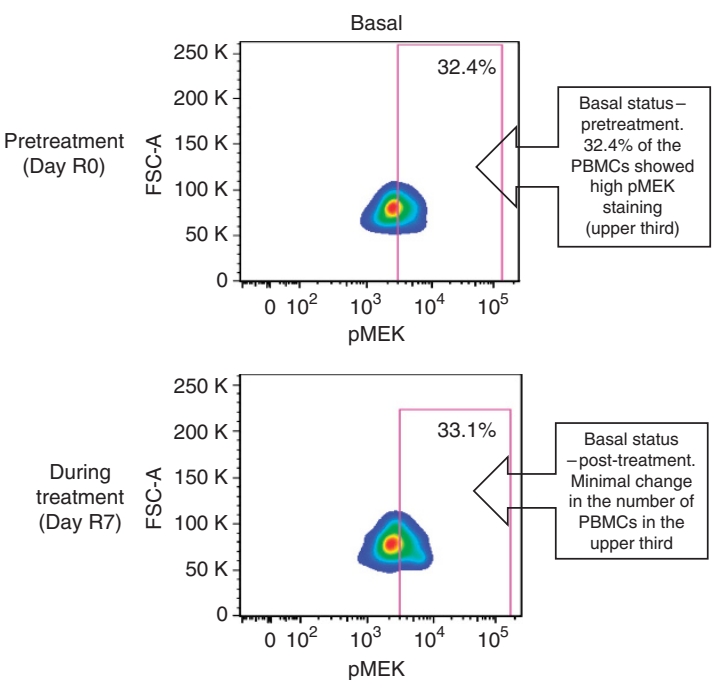

B
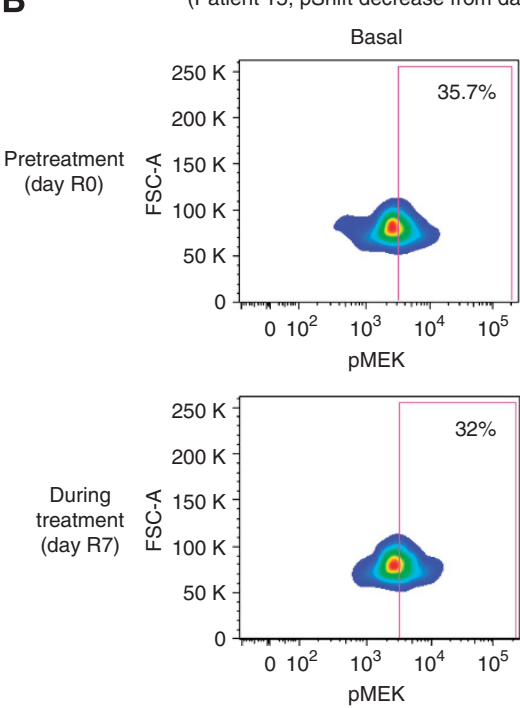

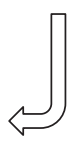

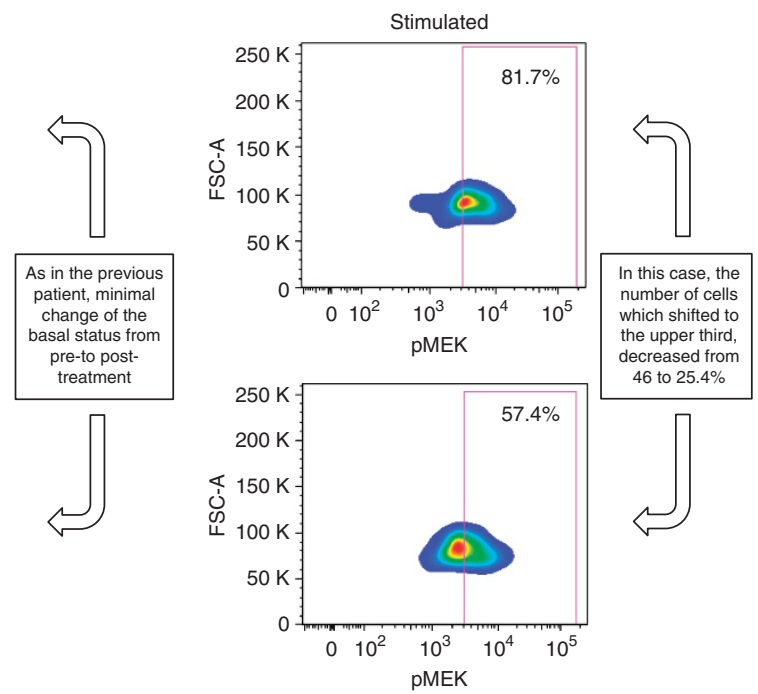

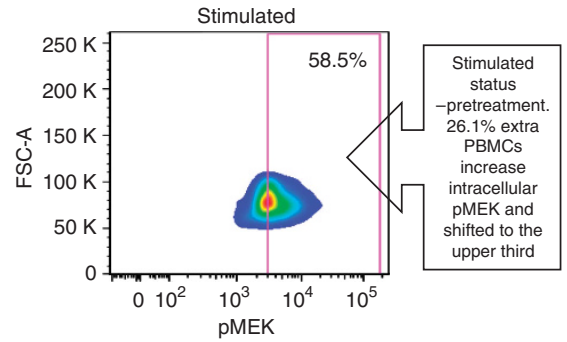

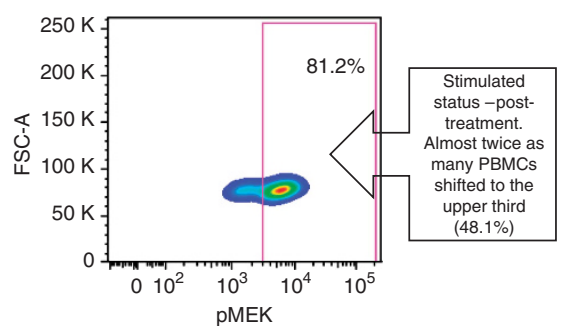

pMEK

0 to 7 , 'negative result', deceased 5 months after starting treatment)

Figure 1. Flow cytometry charts showing the two types of pShift behaviour observed from day R0 to day R7. (A) Shows the phospho-MEK1/2 intensity in PBMCs before (upper charts) and after (lower charts) 7 days of treatment. The left and right charts, respectively, represent the phospho-MEK1/2 staining in vehicle (basal)- and IL3-stimulated samples. In the two pairs of samples shown in A, a larger percentage of monocytes shift to the upper PMEK quadrant in response to stimulation with IL3 in day R7 compared with day RO, what constitutes a positive response. Conversely, the two pairs of samples shown in B, represent a negative response, as the percentage of monocytes shifting to the upper pMEK quadrant is lower in day R7 than in day RO. Of note, the percentage of monocytes in the upper pMEK third in non-stimulated conditions virtually does not vary from patient to patient or from day RO to R7, supporting the need to study the stimulated status.

started on sorafenib $200 \mathrm{mg}$ b.i.d. plus a fixed daily dose of oral cyclophosphamide (50 mg q.d.), and a second pShift test and toxicity assessment were obtained after 7 days of treatment (day R7). In the absence of toxicity, the sorafenib dose was escalated to $400 \mathrm{mg}$ b.i.d. If using this dose, a patient showed $>90 \%$ decrease in pShift, the run-in phase would end and the treatment phase started. Otherwise, sorafenib dose-escalation rounds were repeated every 2 weeks one or more of the three above-mentioned criteria were met. Cyclophosphamide dose was not escalated or modified during the run-in period. After the individual dose-titration of sorafenib, patients then began the treatment phase (cycle 1 day 1 ) of continuous administration of sorafenib plus cyclophosphamide in 28-day cycles.

Objective response was evaluated following RECIST 1.0 criteria (Therasse et al, 2000), after the first cycle and then every two cycles subsequently. Toxicity was evaluated weekly during the run-in phase, bi-weekly in the first cycle, and every 4 weeks subsequently, using Common Terminology Criteria for Adverse Events version 3.0 (CTCAE v3.0) (Cancer Therapy Evaluation Program, National Cancer Institute, Bethesda, MD, USA). Blood pressure was recorded at all pharmacokinetic (PK) sampling time points during the run-in phase and on each subsequent scheduled clinic visit.

Additional rules for dose reduction that were applied during the treatment phase included the following: (1) two 25\% reductions were allowed for sorafenib during the study after the run-in phase; (2) sorafenib monotherapy was allowed, but cyclophosphamide was not to be given as a single-agent; (3) cyclophosphamide was discontinued if any toxicity occurred mandating its dose modification, and no cyclophosphamide dose reductions were planned; (4) if a dose reduction for sorafenib was required during the run-in phase due to toxicity, the rules depicted in Figure 2 were followed.

A Simon's two-stage optimal design $(\mathrm{p} 0=0.05 ; \mathrm{p} 1=0.2$; alpha/ beta errors $=0.05 / 0.1$, respectively) was adopted using the primary 


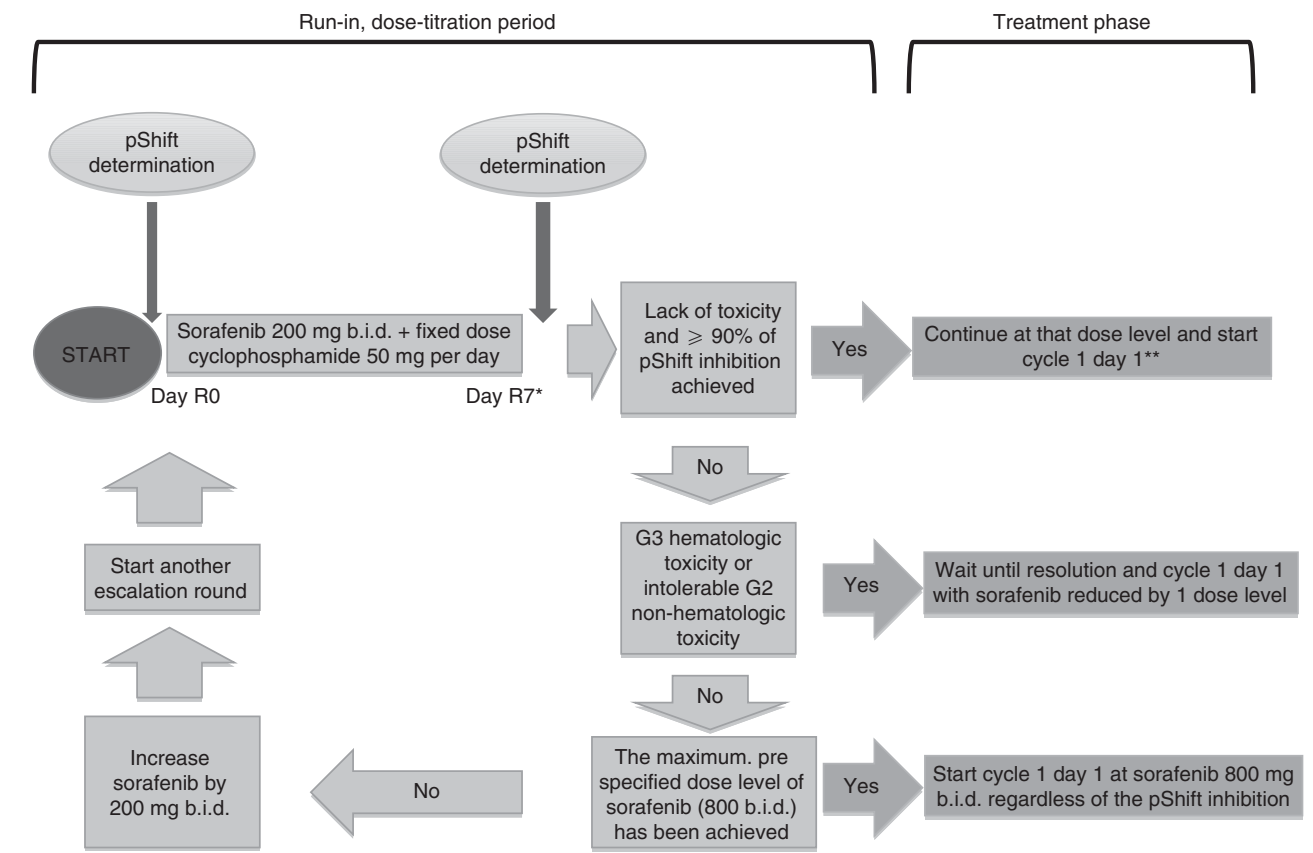

Figure 2. Rules for individual dose-escalation of sorafenib. Schema of sorafenib dose-titration rules. The first pShift within 7 days to the first sorafenib dose ('day $\mathrm{RO}^{\prime}$ ). The escalation rounds were terminated and treatment phase cycle 1 day 1 started as soon as any of the depicted criteria was met, whichever occurred first: (1) reaching a $\geqslant 90 \%$ pShift inhibition; (2) any grade 3 or any grade 2 non-tolerable, non-haematologic toxicity at least possibly related to sorafenib; (3) a maximum dose of $800 \mathrm{mg}$ b.i.d of sorafenib. The following exceptions apply for the dose-titration phase: *After the first round, all the subsequent rounds lasted 14 days. ${ }^{*}$ In the absence of prespecified toxicity, dose-escalation of sorafenib to $400 \mathrm{mg}$ b.i.d. was mandatory after the first round regardless of the pShift.

efficacy end point, which was objective response rate (ORR). A second stage was to be opened if $\geqslant 1 / 21$ responses were observed in the first stage. The regimen would be considered for further evaluation if $\geqslant 4 / 41$ responses were observed in the two stages combined.

Pharmacokinetics. A single blood sample for determining plasma concentration of sorafenib and the M2-N-oxide metabolite was obtained at each time point for PBMC collection for pShift assay during the run-in phase and on cycle 1 day 15 , at $3-5 \mathrm{~h}$ after the morning dose; that is, at appropriately Tmax of sorafenib (Carter, 2004). Pharmacokinetic analysis for sorafenib was done by Bayer HealthCare Pharmaceuticals using a validated liquid chromatography-mass spectrometry method (Moore et al, 2005).

Efficacy evaluation and statistical analysis. The efficacy end points of the trial were overall response rate, progression-free survival (PFS) and overall survival (OS). Candidate prognostic/ predictive markers were changes in pShift (pShift response) and changes in blood pressure (Ryanne Wu et al, 2009; Goodwin et al, 2010; Jubb and Harris, 2010; De Stefano et al, 2011; Rini et al, 2011) using log-rank test and Kaplan-Meier plots. For prognostic/ predictive analyses, PFS and OS were used as the dependent variables, given the expected low ORR. A Cox proportional hazards model was used to evaluate the effects of pShift and tumour grade on PFS and OS.

According to the REMARK criteria for prognostic/predictive factors (McShane et al, 2005), all of the assayed patients were included in the analysis, and the assay was performed prospectively during patient recruitment in a blinded fashion before gathering clinical outcome knowledge. No case had missing data for the tested variables. Technicians involved in performing the assay were blinded to clinical outcome.

Interaction between sorafenib dose, sorafenib $\mathrm{PK}$, and pShift response was assessed using the Spearman's correlation analysis. The influence of sorafenib dose, PK, or pShift response on sorafenib dose reductions and on the incidence of grade 3 or worse adverse events deemed at least possibly related to sorafenib were explored by a multinomial logistic regression analysis. SAS 9.2 software (SAS Institute Inc., Cary, NC, USA) was used.

\section{RESULTS}

Patients and recruitment. From January 2008 to October 2010, 22 patients entered the study. Eighteen had a diagnosis of advanced carcinoid tumour (82\%) and four had advanced islet cell carcinoma (18\%). Median age was 58 years (range: $32-81$ ), and 12 patients were male (55\%). The ECOG performance status was 0 in $7(32 \%)$ and 1 in $15(68 \%)$ patients. The tumour grade according to the Ki67 index was 1,2 , or 3 in $0,11(50 \%)$ and $4(18 \%)$ patients, respectively, and was not evaluated in 7 (32\%). Exposure to prior systemic treatments was $0,1,2,3$ or 7 lines in $8(36 \%), 10(45 \%)$, 2 (9\%), 1, and 1 patient, respectively. Finally, 12 patients (55\%) were on concurrent treatment with somatostatin analogues at study registration.

Sorafenib dose-titration. Of 22 patients, three did not proceed to the treatment phase: one due to grade 4 lipase elevation, one due to clinical deterioration, and one due to poor compliance during the run-in period. The sorafenib dose on cycle 1 day 1 was defined by criteria 1,2 , or 3 in 13,2 , and 1 patients, respectively (59\%, $9 \%$ and $4.5 \%)$. In three patients, the starting dose on cycle 1 day 1 , did not comply with any of the preset criteria: one patient decided not to escalate dose, and two were not escalated by investigator's decision despite toxicity reasons not reaching $\geqslant$ intolerable grade 2 . The starting doses of sorafenib on cycle 1 day 1 are shown for all patients in Table 1.

Safety. Adverse events occurring in $\geqslant 5 \%$ of treatment cycles, deemed to be at least possibly related to the study regimen, are summarised in Table 2 . Grade 3 toxicity was observed in seven patients, and intolerable Grade 2 events in nine patients. There was one grade, four adverse toxic event in two patients (lipase 
Table 1. Summary of pharmacodynamic measurements and dose modifications

Run-in period

\begin{tabular}{|c|c|c|c|c|c|c|c|c|}
\hline Patient & $\begin{array}{l}\text { pShift } \\
\text { d0 }\end{array}$ & $\begin{array}{l}\text { pShift } \\
\text { d7 }\end{array}$ & $\begin{array}{c}\text { pShift } \\
\text { change }(\%)^{a}\end{array}$ & $\begin{array}{l}\text { BP change } \\
(\mathrm{mm} \mathrm{Hg})^{\mathrm{b}}\end{array}$ & $\begin{array}{l}\text { Sorafenib total } \\
\text { daily dose on } \\
\text { C1 d } 1^{c}(\mathrm{mg})\end{array}$ & $\begin{array}{c}\text { Cycle(s) in } \\
\text { which dose } \\
\text { was modified }\end{array}$ & $\begin{array}{l}\text { Final total } \\
\text { daily dose } \\
\qquad(\mathrm{mg})\end{array}$ & $\begin{array}{l}\text { Reason(s) } \\
\text { for dose } \\
\text { modification }\end{array}$ \\
\hline 1 & 27.46 & 13.50 & -50.8 & 4.7 & $400^{1}$ & - & 400 & \\
\hline 2 & 11.96 & -0.36 & -103 & 13.3 & 800 & - & 800 & \\
\hline 3 & 26.46 & 33.80 & 27.7 & -7 & 1200 & $\begin{array}{l}2 \\
2\end{array}$ & $\begin{array}{l}800 \\
400\end{array}$ & $\begin{array}{l}\text { G2 Hand-foot syndrome } \\
\text { (HFS) (intolerable) } \\
\text { Mucositis, diarrhoea, } \\
\text { gas (all intolerable G2) }\end{array}$ \\
\hline 4 & 40.23 & 37.83 & -5.97 & 9.3 & 1600 & $\begin{array}{l}3 \\
3\end{array}$ & $\begin{array}{r}1200 \\
800\end{array}$ & $\begin{array}{l}\text { G3 HFS } \\
\text { G3 HFS }\end{array}$ \\
\hline 5 & 17.80 & 9.63 & -45.9 & -3 & 800 & 8 & 400 & G2 Fatigue \\
\hline 6 & 32.80 & 44.06 & 34.4 & -0.7 & $800^{2}$ & 13 & 400 & G2 Diarrhoea (intolerable) \\
\hline 7 & 46.66 & 53.46 & 14.7 & 1 & $400^{3}$ & 2 & $200 *$ & G2 HFS (intolerable) \\
\hline 8 & 47.66 & 28.13 & -40.9 & 11.7 & 1200 & - & 1200 & \\
\hline 9 & 36.96 & 33.93 & -8.2 & 21.7 & $400^{4}$ & - & 400 & \\
\hline 10 & 51.66 & 33.37 & -35.4 & -2.3 & 800 & 1 & 400 & $\begin{array}{l}\text { G2 Nausea, vomiting } \\
\text { and leg pain }\end{array}$ \\
\hline 11 & 53.73 & 38.00 & -29.3 & 9 & $800^{5}$ & - & 800 & \\
\hline 12 & 12.20 & 2.23 & -81.7 & 5.3 & 800 & - & 800 & \\
\hline 13 & 11.23 & 19.95 & 77.6 & 12.3 & $400^{6}$ & 4 & $200 *$ & G2 Proteinuria ${ }^{\mathrm{e}}$ \\
\hline 14 & 60.13 & 18.67 & -12.1 & -2 & 800 & - & 800 & \\
\hline 15 & 48.33 & 27.40 & -43.3 & 15.7 & $800^{7}$ & - & 800 & \\
\hline 16 & 60.13 & 29.75 & -50.5 & -83.7 & 800 & - & 800 & \\
\hline 17 & 51.00 & 11.57 & -77.3 & 5 & - & - & - & \\
\hline 18 & 47.73 & 58.53 & 22.6 & -1 & 800 & - & 800 & \\
\hline 19 & 29.06 & 10.30 & -64.6 & 23 & - & - & - & \\
\hline 20 & 58.03 & 62.93 & 8.4 & 16.3 & $800^{8}$ & 3 & 400 & G2 Diarrhoea (intolerable) \\
\hline 21 & 57.56 & 25.1 & -56.4 & -75 & 800 & - & 800 & \\
\hline 22 & 48.00 & 43.68 & -9 & 8.7 & - & - & - & \\
\hline
\end{tabular}

Abbreviations: $\mathrm{BP}=$ blood pressure; $\mathrm{pShift}=$ phosphoshift assay

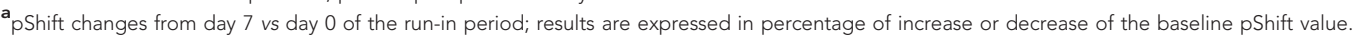

${ }^{\mathbf{b}}$ Average blood pressure on day 7 of run-in phase minus average blood pressure on day 0 of run-in phase (in $\mathrm{mm} \mathrm{Hg}$, calculated as follows: average blood pressure $=$ (systolic blood pressure $+2 \times$ diastolic blood pressure)/3).

${ }^{c}$ Cycle 1 day 1 dose, all cases b.i.d. During the run-in period, doses were modified due to the following reasons: 1: G3 vomiting; G2 rash (intolerable); 2: G3 HFS; 3: G2 HFS (intolerable); 4: G2 diarrhoea (intolerable); 5: G3 AST and ALT elevation; 6: G3 HFS; 7: G2 mucositis and fatigue (intolerable); 8: G3 AST and ALT elevation.

$\mathbf{d}_{\text {In two daily takes except in * (one daily take). }}$

e Proteinuria was $3.0 \mathrm{~g}$ per $24 \mathrm{~h}$, but was sustained over several cycles and dose was reduced by investigator's decision. elevation, one of whom had concurrent grade 4 amylase increase). Twelve patients $(55 \%)$ required a dose reduction of sorafenib, the reasons for dose reductions and the cycles at which they occurred are depicted in Table 1.

Efficacy. A total of 160 treatment cycles were administered. The median number of cycles per patient was 3 (range: $1-38+$ ). All patients were evaluable for PFS and OS. Nineteen patients were evaluable for response. Best response were: 1 partial response (5.3\%) and 13 disease stabilisation (68.4\%), respectively. Median PFS was 3 months (95\% CI: 2-10.7), and 6-month progression-free rate was $46 \%$. Regarding OS, the median duration of OS was 11.7 months (95\% CI: 4.3 months-upper limit not reached), and the 1 -year survival rate was $45 \%$.

Associations between pShift changes and treatment efficacy or toxicity. Upon sorafenib treatment, six patients (27\%) experienced a pharmacodynamic pShift change that was classified as positive, while 16 showed a pShift change that was classified as negative. The individual pShift response and blood pressure changes from day R0 to day R7 of the run-in phase are provided in Table 1.

Median PFS in patients with a negative pShift result was 2.8 months (95\% CI: 1.8-6.7 months), while in patients with a positive pShift result PFS was 14.9 months (95\% CI: 3 months-not reached). Compared with patients that had a negative pShift result, those patients with a positive pShift result had an improvement in PFS that was statistically significant $(P=0.047)$ (Figure 3A).

Median OS for patients with a negative pShift was 6.4 months (95\% CI: 2.8-6.4 months), compared with 21.3 months in patients with a positive pShift result (95\% CI: 7.9 months-not reached). Patients with a positive pShift had an improvement in OS that did not reach statistical significance $(P=0.178)$. Interestingly, all five patients that showed disease progression in their first evaluation ( $\leqslant 8$ weeks of treatment) exhibited a negative pShift result. We also used the Spearman's Rho correlation coefficients to calculate the 
correlation between pShift change from R0 to R7 and PFS and OS. The correlation between pShift and PFS was $0.59(P=0.036)$ and between pShift and OS was $0.72(P<0.001)$, respectively, both correlations being significant. However, when tumour grade was entered in a Cox model along with pShift, the significance of the association between pShift, and PFS and OS is lost $(P=0.259$ and $P=0.099$, respectively).

Table 2. Adverse events occurring in $\geqslant 5 \%$ of the 160 treatment cycles administered, deemed possibly, probably, or definitely related to study treatment

\begin{tabular}{|c|c|c|c|c|}
\hline Event & $\begin{array}{c}\text { Number } \\
\text { of } \\
\text { patients, } \\
\text { any grade }\end{array}$ & $\begin{array}{c}\text { Worst } \\
\text { grade, } \\
\text { any } \\
\text { patient }\end{array}$ & $\begin{array}{l}\text { Number } \\
\text { of cycles, } \\
\text { grade } 3-4\end{array}$ & $\begin{array}{l}\text { Number of } \\
\text { cycles, any } \\
\text { grade }\end{array}$ \\
\hline Hand-foot syndrome & $11(50 \%)$ & 3 & $4(2.5 \%)$ & 100 (62\%) \\
\hline Taste alterations & $5(22 \%)$ & 2 & $0(0 \%)$ & $67(42 \%)$ \\
\hline Hypertension & $3(14 \%)$ & 3 & $29(18 \%)^{a}$ & $64(40 \%)$ \\
\hline Fatigue & $9(41 \%)$ & 3 & $1(0.6 \%)$ & 63 (39\%) \\
\hline Diarrhoea & $13(59 \%)$ & 3 & $3(1.8 \%)$ & $52(32 \%)$ \\
\hline Alopecia & $4(18 \%)$ & 2 & $0(0 \%)$ & $50(31 \%)$ \\
\hline Hypophosphatemia & $11(50 \%)$ & 3 & $6(3.7 \%)$ & $42(26 \%)$ \\
\hline Rash & $9(41 \%)$ & 2 & $0(0 \%)$ & $32(20 \%)$ \\
\hline Lymphopenia & $7(32 \%)$ & 2 & $4(0 \%)$ & $31(19 \%)$ \\
\hline Proteinuria & $6(27 \%)$ & 3 & $1(0.6 \%)$ & $29(18 \%)$ \\
\hline Weight loss & $6(27 \%)$ & 2 & $0(0 \%)$ & $28(18 \%)$ \\
\hline $\begin{array}{l}\text { Aspartate } \\
\text { aminotransferase } \\
\text { elevation }\end{array}$ & $6(27 \%)$ & 3 & $2(1.2 \%)$ & 27 (17\%) \\
\hline Nausea & $8(36 \%)$ & 2 & $0(0 \%)$ & $24(15 \%)$ \\
\hline Thrombocytopenia & $3(14 \%)$ & 1 & $0(0 \%)$ & $16(10 \%)$ \\
\hline Lipase elevation & $7(32 \%)$ & 4 & $2(1.25 \%)$ & $12(7.5 \%)$ \\
\hline $\begin{array}{l}\text { Peripheral sensory } \\
\text { neuropathy }\end{array}$ & $1(4.5 \%)$ & 1 & $0(0 \%)$ & $11(7 \%)$ \\
\hline Amylase elevation & $6(27 \%)$ & 4 & $2(1.2 \%)$ & 9 (6\%) \\
\hline Vomiting & $5(23 \%)$ & 3 & $1(0.6 \%)$ & $8(5 \%)$ \\
\hline $\begin{array}{l}\text { Alanine } \\
\text { aminotransferase } \\
\text { elevation }\end{array}$ & $3(14 \%)$ & 3 & $1(0.6 \%)$ & 7 (5\%) \\
\hline Hypocalcemia & $3(14 \%)$ & 1 & $0(0 \%)$ & $7(5 \%)$ \\
\hline \multicolumn{5}{|c|}{$\begin{array}{l}{ }^{a} \text { A single patient accounted for the } 29 \text { cycles where grade } 3 \text { hypertension was recorded } \\
\text { (requiring two drugs for blood pressure control as per CTCAE v.3.0). }\end{array}$} \\
\hline
\end{tabular}

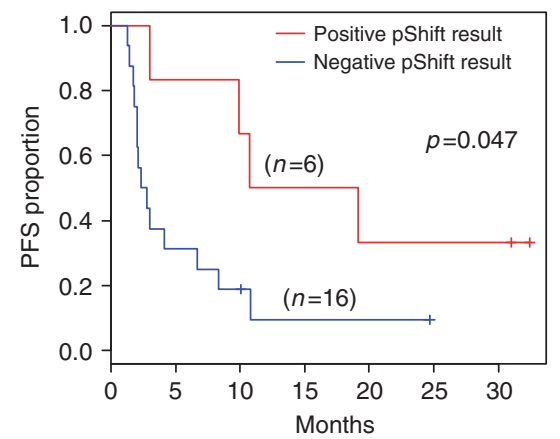

With respect to the relationship between pShift response and toxicity, two multinomial logistic regression models were run. The first model selected the number of sorafenib dose reductions as the dependent variable, while the second model selected the occurrence of grade $\geqslant 3$ adverse events at least possibly related to sorafenib as the dependent variable. The tested predictive (independent) variables included: pShift change (\%) from R0 to R7, sorafenib trough plasma levels, its active metabolite M2-Noxide trough plasma levels on R7 of run-in phase, and sorafenib dose on cycle 1 day 1 . No statistical significance was achieved for any of the variables/models.

Associations between blood pressure changes or PK parameters and efficacy. Blood pressure increases which occurred during treatment and did not achieve statistical significance when correlated with clinical outcome in the current study. For average blood pressure change that occurred between R0 and R7 of run-in phase, measured in $\mathrm{mm} \mathrm{Hg}$, Cox univariate analysis yielded hazard ratios for OS of 0.98 (95\% CI: $0.96-1.01 ; P=0.13)$ and PFS of 0.99 (95\% CI: $0.97-1.02 ; P=0.524$ ), respectively. The individual blood pressure changes from R0 to R7 of the run-in phase are provided in Table 1.

Plasma levels of sorafenib on R7 and day 21 of run-in phase, and cycle 1 day 15 are described in Supplementary Figure 1. Neither sorafenib dose on cycle 1 day 1 , nor sorafenib or M2-Noxide metabolite trough plasma levels on R7 of run-in phase, predicted pShift behaviour. Considering pShift change from R0 to R7 as a continuous variable ranging from $-100 \%$ to $+100 \%$, the correlations (Rho) between sorafenib levels and pShift, and M2-Noxide metabolite levels and pShift were 0.02 (95\% CI: $-0.45-0.48$; $P=0.94)$ and 0.14 (95\% CI: $-0.35-0.57 ; P=0.576)$, respectively. Accordingly, sorafenib dose on cycle 1 day 1 did not correlate with PFS (Rho $=-0.005 ; 95 \%$ CI: $-0.46-0.45 ; P=0.984)$ or OS (Rho $=0.05 ; 95 \%$ CI: $-0.41-0.45 ; P=0.84)$.

\section{DISCUSSION}

Therapeutic progress in the treatment of advanced NET has been achieved in the past few years. Three recently reported phase III trials have shown clinical benefit using sunitinib (Raymond et al, 2011), everolimus (Yao et al, 2011), or single-agent octreotide (Rinke et al, 2009). Currently, no validated biomarkers exist that would enable the optimal selection of systemic therapy for these patients. Although the demonstration of pharmacodynamic target modulation that offers proof-of-mechanism may not be sufficient, as there are likely intrinsic individual tumour- and host-related determinants that affect treatment response to any targeted agent, an early read-out of pharmacodynamic effects may offer a prognostic/predictive measure of treatment outcome.

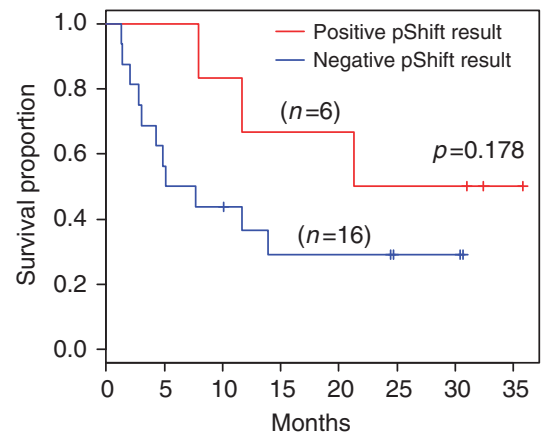

Figure 3. pShift and efficacy. Kaplan-Meier plots for PFS (left) and OS (right) in patients with a positive result in the pShift ( $n=6$ ) vs patients with a negative result $(n=16)$. 
Furthermore, the assessment of the dynamic status of a signalling pathway can potentially be more informative of a drug's pharmacodynamic effects than a basal status (Irish et al, 2004; Sachs et al, 2005). In order to evaluate the status of the RAF isoforms that are targets of sorafenib, we developed an in vivo flow cytometry-based PBMC assay (pShift) that was incorporated in the design of the SORNET clinical trial in patients with advanced NET to provide individual sorafenib dose-titration and to determine its prognostic/ predictive value. In a previous pilot study of seven patients with different advanced malignancies enrolled in an expansion cohort of a phase I targeted combination trial of sorafenib plus erlotinib (Quintela-Fandino et al, 2010), we had observed variable degrees of pShift decrease and consequently designed the sorafenib dosetitration process in the current SORNET trial to pursue maximum pShift decrease. Although pShift increases were detected in patients enrolled in the SORNET trial, we decided not to alter the preset dosetitration rules, as it was not clear at that time what constituted a favourable biological response. During the course of the SORNET trial, the published findings by Rosen and Poulikakos (Poulikakos et al, 2010) enabled a more meaningful interpretation of the pShift response. They suggest that, at pharmacologic doses, in tissues with mutant b-RAF and wild-type RAS, pan-RAF inhibitors such as sorafenib effectively block RAS-RAF-MEK signalling. However, in tissues with wild-type RAS and RAF background (such as in PBMCs), pan-RAF inhibitors can trans-activate c-RAF and enhance MEK activation. Hence, an increase in MEK phosphorylation (pShift increase or a positive value) when measured in PBMCs would represent a favourable biological proof of pharmacodynamic effects of sorafenib. Among the 22 patients assayed, none of them showed appreciable differences in the basal status of pMEK from R0 to R7 (data not shown). Thus, basal assessment of biomarkers of a putative signalling pathway may not be relevant clinically, and dynamic assessment should be obtained whenever it is technically feasible. Finally, in our study, pShift responses to sorafenib exposure did not add to conventional toxicity-guided dose-titration.

Indeed, despite the small sample size in the SORNET trial, we observed both positive and negative pShift results as an early pharmacodynamic read-out, and these values correlated with the clinical course. The validation of this observation in larger studies in advanced NET, or ideally in other malignancies in which sorafenib already has a proven efficacy in unselected patients, would enable the determination whether the pShift response can be a useful early predictive biomarker for patient selection. However, in the absence of a control group, it is not possible to ascertain whether the pShift is a prognostic biomarker of clinical outcome regardless of treatment $v s$ a predictive biomarker specific for sorafenib efficacy.

Our results with pShift are consistent with the premise that the antitumour effect of an agent correlates with its pharmacodynamic effect on its molecular targets. A limitation of our study is that MEK activation can be a surrogate marker of VEGFR2 and PDGFR-B inhibition, especially in NET, which often exhibits wildtype RAF and RAS. Indeed, because of the technical reasons mentioned in the introduction, the only way to demonstrate target engagement in PBMCs was to measure MEK activation, rather than VEGFR2 or PDGFR-B. Thus, it is impossible to ascertain whether RAF engagement reflects VEGFR2/PDGFR-B engagement and is an adequate surrogate biomarker of the putative targets of sorafenib in NET, or not. To assess this hypothesis, paired tumour biopsies that would allow one to study in tumour tissue the changes of RAF or pVEGFR/pPDGFR signal transduction would be needed, but real-time dynamic signal transduction studies are not technically feasible in tumour tissue samples yet. Recently, RAF stimulation in healthy tissue has been proposed as a mechanism for side effects from RAF inhibitors, such as keratoacanthomas (Oberholzer et al, 2012; Su et al, 2012), although it is not known whether such stimulation occurs universally in every patient upon exposure to RAF inhibitors. Our observations suggest that RAF stimulation does not occur basally and is evident upon dynamic assessment, and in addition appears to be restricted to a fraction of the patients upon treatment with sorafenib. Finally, the effects of cyclophosphamide on pShift is unknown, as preclinical studies were not performed with this agent.

It is relevant to highlight that trough sorafenib plasma levels measured in the current study were consistent with those published for sorafenib doses between $200 \mathrm{mg}$ b.i.d. and $400 \mathrm{mg}$ b.i.d. at steady state (Strumberg et al, 2005). It is also interesting to note that the pShift behaviour was not dependent on the sorafenib dose or its plasma concentrations, and that these factors did not correlate with clinical outcome. Our finding is consistent with the published data that the pharmacodynamic effects of RAF inhibitors are determined primarily by the genetic background of the target rather than by drug concentrations (Rajakulendran et al, 2009; Hatzivassiliou et al, 2010; Poulikakos et al, 2010).

Although the pharmacodynamic effects should be correlated with toxicity, in our study, neither the pShift response nor the sorafenib dose at cycle 1 day 1 seemed to be predictive factors of toxicity or of sorafenib dose reduction events. This may be partly explained by the semi-quantitative nature of toxicity scales, which constitutes a challenge to find statistical associations. A similar explanation could apply to blood pressure changes as a potential predictive factor for antiangiogenic therapy (Ryanne Wu et al, 2009; Goodwin et al, 2010; Jubb and Harris, 2010; De Stefano et al, 2011; Rini et al, 2011). Although the largest published study on the association between blood pressure rise and clinical benefit is retrospective and not preplanned (Rini et al, 2011), a positive relationship has been suggested. Our data identified a trend, but it did not achieve statistical significance.

The SORNET trial was terminated after 22 patients were enrolled due to the modest activity of the study combination of dose-titrated sorafenib with metronomic cyclophosphamide in indirect inter-trial comparisons. These include, for example, a large phase II trial of single-agent sorafenib at $400 \mathrm{mg}$ b.i.d in 93 advanced NET patients in which an ORR of $10 \%$ was demonstrated (Hobday et al, 2007). The 5.3\% obtained in the SORNET trial seemed insufficient to pursue the combination further without patient selection.

In conclusion, the pShift assay evaluating in vivo RAF signal transduction was associated with treatment efficacy in patients with NET treated with sorafenib plus cyclophosphamide. Pharmacodynamic assessment of treatment effect on a molecular target, such as pShift, may be a biomarker of sensitivity for sorafenib and other RAF inhibitors, and could potentially be utilised as a tool for patient selection. The current results must be interpreted with caution as they are based on the pharmacodynamic response observed in only a very limited sample size $(n=6$ patients with positive pShift). Further validation of the pShift assay in different tumour types is warranted.

\section{ACKNOWLEDGEMENTS}

MQF is a recipient of an ASCO Young Investigator Award (2007), an AECC (Spanish League Against Cancer, 'Beca de retorno'), and a FIS (Ref. Number PI 10/0288 - 'Fondo de Investigación Sanitaria') grant. The SORNET clinical trial was partially funded by Bayer-Onyx Canada.

\section{REFERENCES}

Carter CW C (2004) Sorafenib Investigator Brochure V.5. Bayer Pharmaceuticals Corporation.

Carter CW C (2010) Sorafenib Investigator Brochure V.11.0. Bayer Pharmaceuticals Corporation. 
De Stefano A, Carlomagno C, Pepe S, Bianco R, De Placido S (2011) Bevacizumab-related arterial hypertension as a predictive marker in metastatic colorectal cancer patients. Cancer Chemother Pharmacol 68(5): $1207-1213$

Goodwin R, Ding K, Seymour L, LeMaitre A, Arnold A, Shepherd FA, Dediu M, Ciuleanu T, Fenton D, Zukin M, Walde D, Laberge F, Vincent M, Ellis PM, Laurie SA (2010) Treatment-emergent hypertension and outcomes in patients with advanced non-small-cell lung cancer receiving chemotherapy with or without the vascular endothelial growth factor receptor inhibitor cediranib: NCIC Clinical Trials Group Study BR24. Ann Oncol 21: 2220-2226.

Gutierrez ME, Kummar S, Giaccone G (2009) Next generation oncology drug development: opportunities and challenges. Nat Rev Clin Oncol 6: 259-265.

Hanahan D (1985) Heritable formation of pancreatic beta-cell tumours in transgenic mice expressing recombinant insulin/simian virus 40 oncogenes. Nature 315: 115-122.

Hatzivassiliou G, Song K, Yen I, Brandhuber BJ, Anderson DJ, Alvarado R, Ludlam MJ, Stokoe D, Gloor SL, Vigers G, Morales T, Aliagas I, Liu B, Sideris S, Hoeflich KP, Jaiswal BS, Seshagiri S, Koeppen H, Belvin M, Friedman LS, Malek S (2010) RAF inhibitors prime wild-type RAF to activate the MAPK pathway and enhance growth. Nature 464: 431-435.

Hobday TJ, Rubin J, Holen K, Picus J, Donehower R, Marschke R, Maples W, Lloyd R, Mahoney M, Erlichman C (2007) MC044h, a phase II trial of sorafenib in patients (pts) with metastatic neuroendocrine tumors (NET): A Phase II Consortium (P2C) study. J Clin Oncol 18S: 4504.

Irish JM, Hovland R, Krutzik PO, Perez OD, Bruserud O, Gjertsen BT, Nolan GP (2004) Single cell profiling of potentiated phospho-protein networks in cancer cells. Cell 118: 217-228.

Jubb AM, Harris AL (2010) Biomarkers to predict the clinical efficacy of bevacizumab in cancer. Lancet Oncol 11: 1172-1183.

McShane LM, Altman DG, Sauerbrei W, Taube SE, Gion M, Clark GM (2005) Reporting recommendations for tumor marker prognostic studies (REMARK). J Natl Cancer Inst 97: 1180-1184.

Moore M, Hirte HW, Siu L, Oza A, Hotte SJ, Petrenciuc O, Cihon F, Lathia C, Schwartz B (2005) Phase I study to determine the safety and pharmacokinetics of the novel Raf kinase and VEGFR inhibitor BAY 439006, administered for 28 days on/7 days off in patients with advanced, refractory solid tumors. Ann Oncol 16: 1688-1694.

Oberholzer PA, Kee D, Dziunycz P, Sucker A, Kamsukom N, Jones R, Roden C, Chalk CJ, Ardlie K, Palescandolo E, Piris A, Macconaill LE, Robert C, Hofbauer GF, McArthur GA, Schadendorf D, Garraway LA (2012) RAS mutations are associated with the development of cutaneous squamous cell tumors in patients treated with RAF inhibitors. J Clin Oncol 30: 316-321.

Pietras K, Hanahan D (2005) A multitargeted, metronomic, and maximumtolerated dose 'chemo-switch' regimen is antiangiogenic, producing objective responses and survival benefit in a mouse model of cancer. J Clin Oncol 23: 939-952.

Poulikakos PI, Zhang C, Bollag G, Shokat KM, Rosen N (2010) RAF inhibitors transactivate RAF dimers and ERK signalling in cells with wild-type BRAF. Nature 464: 427-430.

Quintela-Fandino M, Le Tourneau C, Duran I, Chen EX, Wang L, Tsao M, Bandarchi-Chamkhaleh B, Pham NA, Do T, MacLean M, Nayyar R, Tusche MW, Metser U, Wright JJ, Mak TW, Siu LL (2010) Phase I combination of sorafenib and erlotinib therapy in solid tumors: safety, pharmacokinetic, and pharmacodynamic evaluation from an expansion cohort. Mol Cancer Ther 9: 751-760.

Rajakulendran T, Sahmi M, Lefrancois M, Sicheri F, Therrien M (2009) A dimerization-dependent mechanism drives RAF catalytic activation. Nature 461: 542-545.
Raymond E, Dahan L, Raoul JL, Bang YJ, Borbath I, Lombard-Bohas C, Valle J, Metrakos P, Smith D, Vinik A, Chen JS, Horsch D, Hammel P, Wiedenmann B, Van Cutsem E, Patyna S, Lu DR, Blanckmeister C, Chao R, Ruszniewski P (2011) Sunitinib malate for the treatment of pancreatic neuroendocrine tumors. N Engl J Med 364: 501-513.

Rini BI, Cohen DP, Lu DR, Chen I, Hariharan S, Gore ME, Figlin RA, Baum MS, Motzer RJ (2011) Hypertension as a biomarker of efficacy in patients with metastatic renal cell carcinoma treated with sunitinib. J Natl Cancer Inst 103: 763-773.

Rinke A, Muller HH, Schade-Brittinger C, Klose KJ, Barth P, Wied M, Mayer C, Aminossadati B, Pape UF, Blaker M, Harder J, Arnold C, Gress T, Arnold R (2009) Placebo-controlled, double-blind, prospective, randomized study on the effect of octreotide LAR in the control of tumor growth in patients with metastatic neuroendocrine midgut tumors: a report from the PROMID Study Group. J Clin Oncol 27: 4656-4663.

Ryanne Wu R, Lindenberg PA, Slack R, Noone AM, Marshall JL, He AR (2009) Evaluation of hypertension as a marker of bevacizumab efficacy. J Gastrointest Cancer 40: 101-108.

Sachs K, Perez O, Pe'er D, Lauffenburger DA, Nolan GP (2005) Causal protein-signaling networks derived from multiparameter single-cell data. Science 308: 523-529.

Strumberg D, Richly H, Hilger RA, Schleucher N, Korfee S, Tewes M, Faghih M, Brendel E, Voliotis D, Haase CG, Schwartz B, Awada A, Voigtmann R, Scheulen ME, Seeber S (2005) Phase I clinical and pharmacokinetic study of the Novel Raf kinase and vascular endothelial growth factor receptor inhibitor BAY 43-9006 in patients with advanced refractory solid tumors. J Clin Oncol 23: 965-972.

Su F, Viros A, Milagre C, Trunzer K, Bollag G, Spleiss O, Reis-Filho JS, Kong X, Koya RC, Flaherty KT, Chapman PB, Kim MJ, Hayward R, Martin M, Yang H, Wang Q, Hilton H, Hang JS, Noe J, Lambros M, Geyer F, Dhomen N, Niculescu-Duvaz I, Zambon A, Niculescu-Duvaz D, Preece N, Robert L, Otte NJ, Mok S, Kee D, Ma Y, Zhang C, Habets G, Burton EA, Wong B, Nguyen H, Kockx M, Andries L, Lestini B, Nolop KB, Lee RJ, Joe AK, Troy JL, Gonzalez R, Hutson TE, Puzanov I, Chmielowski B, Springer CJ, McArthur GA, Sosman JA, Lo RS, Ribas A, Marais R (2012) RAS mutations in cutaneous squamous-cell carcinomas in patients treated with BRAF inhibitors. $N$ Engl J Med 366: 207-215.

Therasse P, Arbuck SG, Eisenhauer EA, Wanders J, Kaplan RS, Rubinstein L, Verweij J, Van Glabbeke M, van Oosterom AT, Christian MC, Gwyther SG (2000) New guidelines to evaluate the response to treatment in solid tumors. European Organization for Research and Treatment of Cancer, National Cancer Institute of the United States, National Cancer Institute of Canada. J Natl Cancer Inst 92: 205-216.

Yagisawa M, Saeki K, Okuma E, Kitamura T, Kitagawa S, Hirai H, Yazaki Y, Takaku F, Yuo A (1999) Signal transduction pathways in normal human monocytes stimulated by cytokines and mediators: comparative study with normal human neutrophils or transformed cells and the putative roles in functionality and cell biology. Exp Hematol 27: 1063-1076.

Yao JC, Shah MH, Ito T, Bohas CL, Wolin EM, Van Cutsem E, Hobday TJ, Okusaka T, Capdevila J, de Vries EG, Tomassetti P, Pavel ME, Hoosen S, Haas T, Lincy J, Lebwohl D, Oberg K (2011) Everolimus for advanced pancreatic neuroendocrine tumors. N Engl J Med 364: 514-523.

This work is published under the standard license to publish agreement. After 12 months the work will become freely available and the license terms will switch to a Creative Commons AttributionNonCommercial-Share Alike 3.0 Unported License.

Supplementary Information accompanies the paper on British Journal of Cancer website (http://www.nature.com/bjc) 